\title{
Dynadesis for Treatment of Dynamic Scaphoid Instability with 20-Year Results
}

\author{
Houshang Seradge, MD ${ }^{1}$ (i) Winfred Parker, BS $^{1} \quad$ Carrie Seradge, BS, CHT ${ }^{1}$ Cyrus Steppe, BA, MS ${ }^{10}$ \\ Ashley McKenzie, $\mathrm{RT}^{1}$ \\ ${ }^{1}$ Seradge Medical, Oklahoma City, Oklahoma \\ Address for correspondence Houshang Seradge, MD, Seradge \\ Medical, 5501 East, 33rd Street, Edmond, OK 73013 \\ J Wrist Surg 2023;12:73-80. \\ (e-mail: seradge@gmail.com).
}

\begin{abstract}
Background Even though the scapholunate interosseous ligament is the most common wrist ligament injury, its treatment remains a challenge for hand surgeons. We report on a surgical treatment (Dynadesis) for dynamic scaphoid instability (DSI) with a 20-year follow-up period.

Description of Technique Dynadesis utilizes antagonist forearm muscles in order to synergistically provide dynamic stabilization to the scaphoid when the wrist is loaded. It is a tendon-to-tendon transfer with the following two components: 1) Dorsal-The extensor carpi radialis longus (ECRL) is passed through a hole in the reduced, distal scaphoid, providing the scaphoid with an independent extension force. 2) Volar-A dynamic checkrein is created by tension-locking the ECRL tendon around the flexor carpi radialis (FCR) tendon. The portion of the FCR distal to the scaphoid tethers and tightens with contracture of the ECRL and FCR muscles.

\section{Keywords}

- carpal instability

- long term results

- scapholunate ligament

- tenodesis

- wrist instability

- scaphoid

- dynamic scaphoid instability

Patients and Methods Twenty patients ( 21 wrists) were treated with Dynadesis and reevaluated 20 years later (range: $20-27$ years).

Results Average grip strength improved by $8 \mathrm{~kg}$. The average wrist flexion-extension arc decreased by $3^{\circ}$. Wrist X-rays showed no radiocarpal arthritis. On the Mayo wrist score, $81 \%$ reported excellent to good results (average: 89 ). Pain levels improved by $90 \%$, with $76 \%$ of patients reporting no pain. All patients (100\%) were satisfied with their results and would recommend the procedure.

Conclusions Dynadesis is specifically designed for the treatment of DSI. It avoids the eventual complication of osteoarthritis and does not sacrifice wrist motion. A predictable and satisfactory long-term result is obtainable with correct patient selection based on clinical staging and arthroscopic findings.
\end{abstract}

Scaphoid instability is a spectrum of instabilities, ${ }^{1}$ ranging from predynamic ${ }^{2}$ to static and must be treated, based on the pathology and stage of instability. ${ }^{3-6}$ Using a "one treatment fits all" approach has not been acceptable. ${ }^{3,6-8}$ Soft-tissue procedures, such as capsulodesis ${ }^{9,10}$ or tendon grafts, $, 3,11-14$

received

May 13, 2021

accepted after revision

July 27, 2021

article published online

September 28, 2021
DOI https://doi.org/

10.1055/s-0041-1735304.

ISSN 2163-3916. limit scaphoid motion, and the repair ultimately fails by stretching out over time. ${ }^{11,13,15}$ The various boney stabilization procedures $8,16,17$ are biomechanically unsound. $8,18,19$ They are acceptable as salvage procedures for static scaphoid instability (Garcia-Elias IV-VI ${ }^{3}$ ); however, they should not be

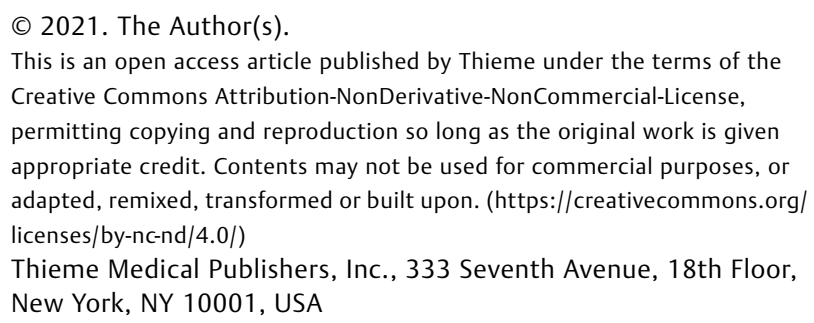




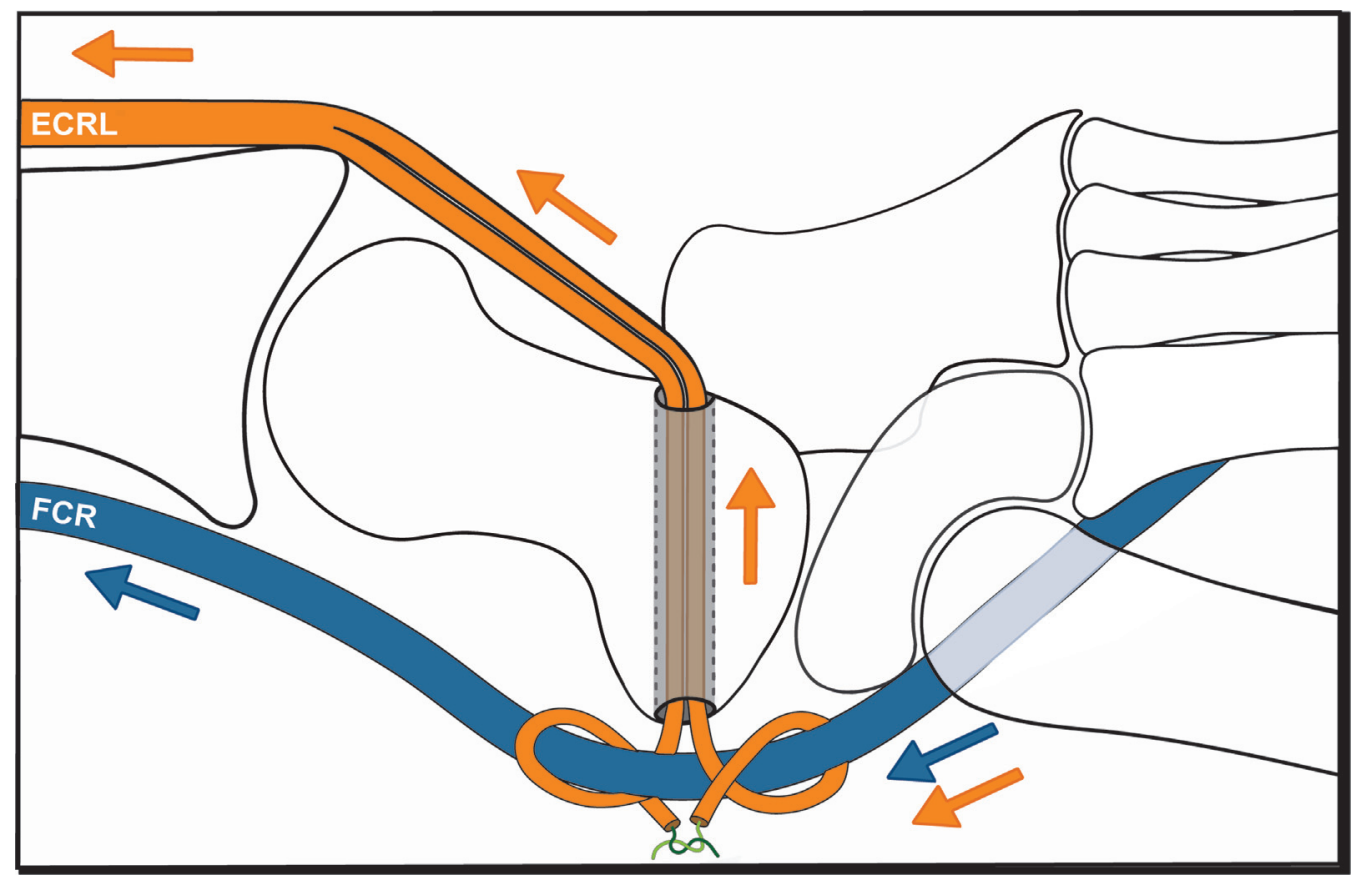

Fig. 1 Dynadesis schematic architecture. Orange arrows: effect of extensor carpi radialis longus (ECRL) on the scaphoid. Blue arrows: effect of flexor carpi radialis (FCR) on the scaphoid. Combined orange and blue arrows: dynamic tethering effect of ECRL and FCR on distal volar scaphoid ligaments.

used to treat dynamic scaphoid instability ${ }^{1}$ (DSI), as they turn a dynamic instability into a fixed deformity. The treatment goal for scaphoid instability is to restore the normal kinematics of the scaphoid. ${ }^{20}$ The available bony and soft-tissue reconstruction procedures do not achieve the goal, resulting in eventual negative outcomes. ${ }^{8,15,18}$

From the spectrum of scaphoid instabilities, dynamic scaphoid instability poses a particular treatment challenge as well as opportunity. Less force is required to stabilize the scaphoid because some of its primary and secondary restraints remain intact. ${ }^{21}$ The scaphoid is stable at rest but cannot maintain its functional and stable position when the wrist is loaded. ${ }^{1,22}$ When the load is removed, the scaphoid returns to its normal state of equilibrium. ${ }^{1,22}$

The scaphoid is devoid of direct muscle attachment; therefore, its functional stability depends on the integrity of its ligaments (passive stabilizers), ${ }^{5,23-26}$ joint reactive forces, ${ }^{27}$ and the forces applied by forearm muscles (active stabilizers). ${ }^{28-32}$ We hypothesized that the dynamic stabilizers of the wrist could be used to stabilize the scaphoid for treatment of DSI. This treatment must encompass the following four criteria: 1) It should avoid limiting scaphoid motion or fixing the scaphoid in one position. 2) It should provide dynamic assistance to extend the scaphoid when the wrist is loaded. 3) It should provide a synchronistic dynamic force to resist scaphoid flexion and augment the volar distal scaphoid ligaments when the wrist is loaded. ${ }^{21,25} 4$ ) It should not stretch over time. ${ }^{15}$

We designed a procedure termed Dynadesis, ${ }^{6,33}$ which would utilize the neuromuscular feedback system. When the wrist is loaded, the dynamic stabilizers of the scaphoid are stimulated by mechanoreceptors in the scapholunate interosseous ligament ${ }^{30}$ (SLIL) within $20 \mathrm{msec}$ and reach active stabilization within $150 \mathrm{msec}^{30}$ They become inactive when the load is removed. This corrective architecture uses the antagonist forearm muscles to apply a synergistic and simultaneous dynamic force to the dorsal and volar scaphoid, in order to resist the forces flexing the scaphoid when the wrist is loaded. The construct has two components (-Fig. 1): (1) Dorsal-The extensor carpi radialis longus (ECRL) tendon passes through a hole of the reduced distal scaphoid to function as a dynamic force that directly extends the scaphoid when the wrist is loaded. (2) Volar-A dynamic checkrein is created by tension-locking the ECRL tendon around the flexor carpi radialis (FCR) tendon that tightens with activation of the ECRL and relaxes when the ECRL relaxes. The portion of the FCR distal to the scaphoid tethers and tightens with cocontraction ${ }^{30}$ of the ECRL and FCR muscles to support the volar distal scaphoid ligaments.

\section{Surgical Technique}

Expose the ECRL tendon through a 4-cm longitudinal incision starting at the base of the 2nd metacarpal. Detach the ECRL tendon from its insertion as distally as possible and retract proximally. Perform a T-capsulotomy to expose the scaphoid. Reduce the scaphoid into full extension and, if necessary, release the radioscaphoid ligaments ${ }^{34}$ and capsule. Fix the scaphoid with two or three $0.45 \mathrm{~K}$-wires inserted at the scapho-trapezio-trapezoid (STT) region, leaving sufficient space in the distal scaphoid for making a tunnel. Although more challenging, superior immobilization of the scaphoid is obtained through the longitudinal axis. Use a burr to make a tunnel in a dorsal-volar direction, perpendicular to the distal scaphoid, and at equal distance from each side of the scaphoid (-Fig. 2). Limit the size of the drill hole to $1 / 3 \mathrm{rd}$ 


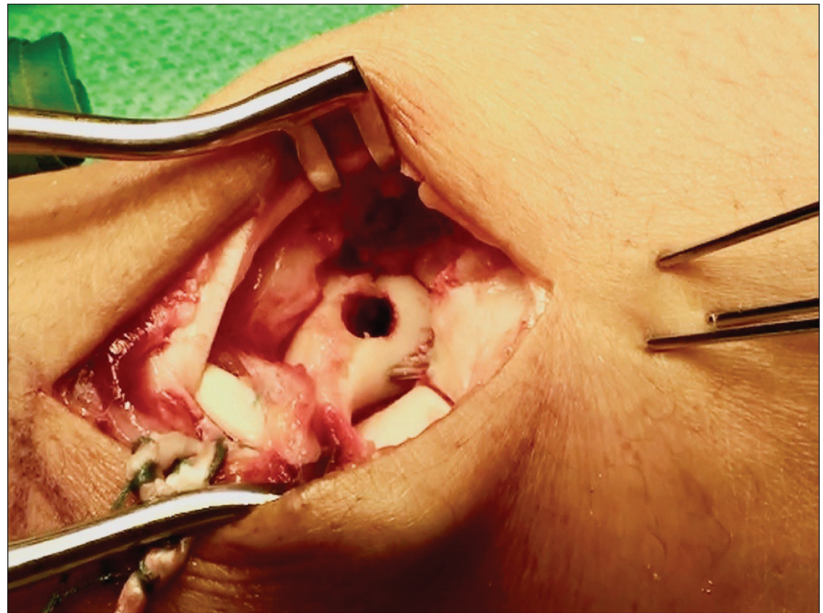

Fig. 2 Drill hole through the distal scaphoid reduced and fixed in extension.

of the width of the distal scaphoid to maintain sufficient osseous walls around the drill hole. If the width of the scaphoid allows, enlarge the diameter of the tunnel to accommodate the thickness of the ECRL tendon. If the tunnel is narrow, use 2/3rd width of the harvested ECRL. Split the harvested ECRL tendon longitudinally into two, 3-cm slips. Use a double-ended, braided, nonabsorbable, number 2-0 suture on the main body of the ECRL, immediately proximal to the ECRL slips. Moving distally, make a running-locking suture on each slip which will strengthen the slips and function as an extension of each slip (-Fig. 3). Thread both sutures into a large, straight Keith needle. Pass the needle through the tunnel, directed radially and distally to avoid the radial artery and median nerve, until it breaks through the skin. Make a 1 to $2 \mathrm{~cm}$ oblique skin incision centered over the needle hole and open the FCR sheath. Retract the FCR and follow the needle to its emerging point from the FCR subsheath and scaphoid. Clean the exit hole by inserting small, closed scissors, and strip the soft tissue from the volar opening of the tunnel in the scaphoid to allow the ECRL slips

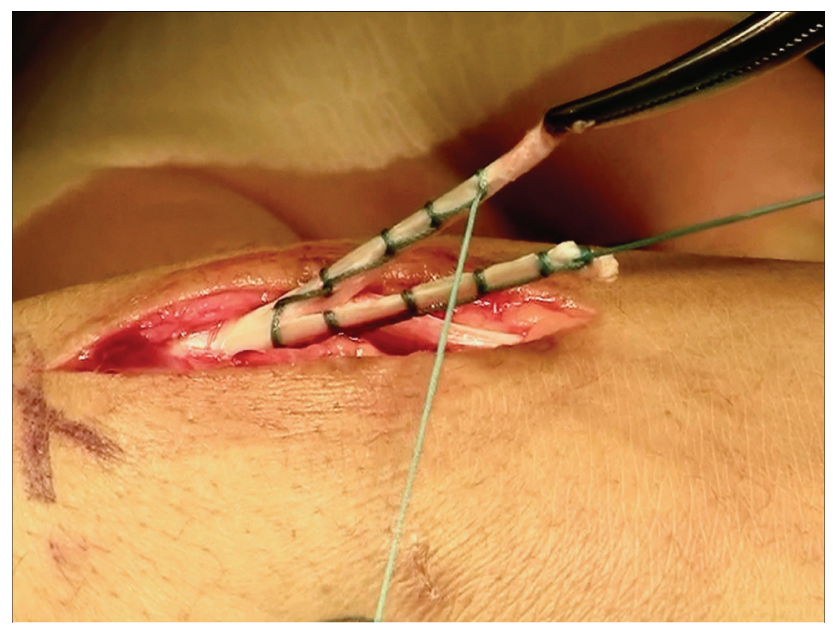

Fig. 3 The slips of extensor carpi radialis longus (ECRL) reinforced and extended by a nonabsorbable, double-ended, running-locking suture.

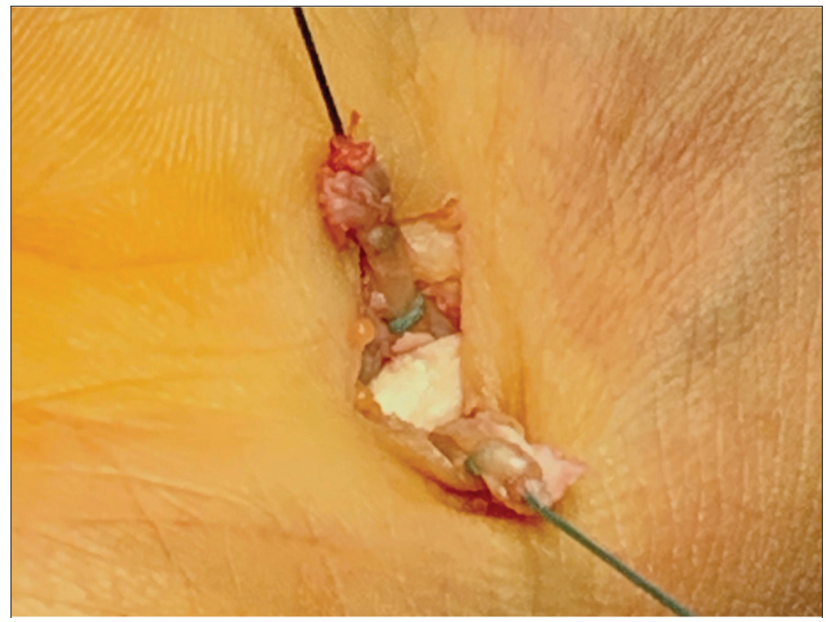

Fig. 4 The extensor carpi radialis longus (ECRL) slips delivered volarly on opposite sides of the flexor carpi radialis (FCR) tendon.

to pass through unimpeded. Pull the needle, sutures and ECRL tendon slips through the scaphoid and deliver each slip to opposite sides of the FCR tendon ( - Fig. 4). Extend the wrist and pull on each slip of the ECRL tendon to make sure it is clear of any impingement and achieves maximum length. Maintain tension on one slip of the ECRL tendon while wrapping the other slip around the FCR. A small, right-angle hemostat facilitates this maneuver. Repeat with the second slip, wrapping it in the opposite direction around the FCR tendon and overlapping the first slip. Maintain the tension on the wrapped slips of ECRL and while pronating the hand to verify that the slips have passed through the tunnel unencumbered. Then, secure the two slips of the ECRL around the FCR by tying the sutures together. This configuration creates a tension-locking mechanism (-Fig. 5), similar to a clove hitch knot, except that the ends are tied together to prevent unraveling. The ECRL is anchored to the FCR without compromising the integrity of the FCR. Verify that the ECRL slips are under tension and that they glide through the scaphoid when pulled dorsally (-Fig. 6). After hemostasis, close the wrist capsule and skin. Immobilize in a thumb spica splint until pin retrieval 6 weeks later. Start rehabilitation to regain range of motion, power, endurance, and proprioception.

\section{Materials and Methods}

Dynamic scaphoid instability was diagnosed in patients with a chief complaint of wrist pain during loading activities with an extended wrist (i.e., flat palm push-ups or pushing off a chair. ${ }^{23}$ ) by clinical examination, X-rays (including a clenched fist X-ray), and arthroscopy. Patients who failed at least 6 months of conservative treatment underwent arthroscopic synovectomy and debridement, evaluation of joint surfaces, and classification of the SLIL tear. ${ }^{35,36}$ Arthroscopic repair of the SLIL was not attempted for any of these patients, because their conditions were chronic. Patients underwent the Dynadesis procedure if they did not respond to arthroscopic debridement, remained symptomatic, and 


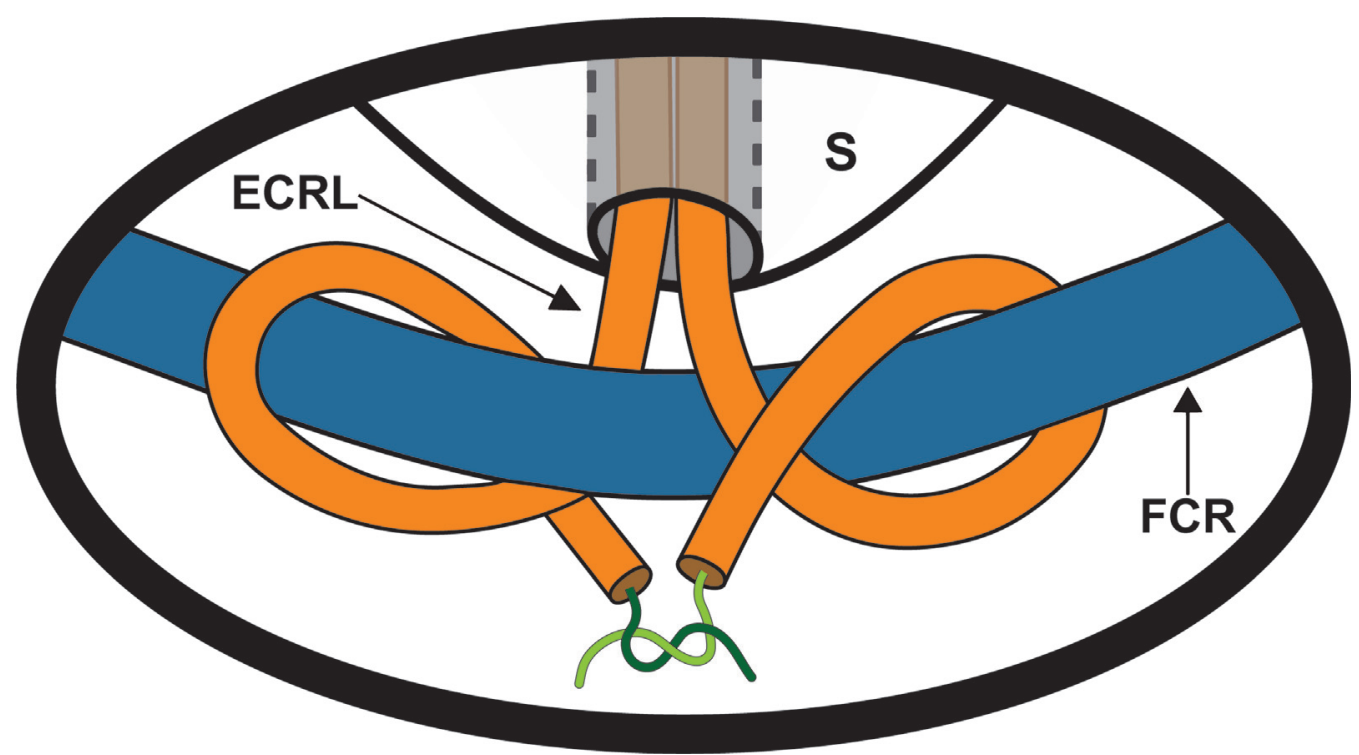

Fig. 5 Schematic of tension locking suture mechanism. ECRL, extensor carpi radialis longus; FCR, flexor carpi radialis; S, scaphoid.

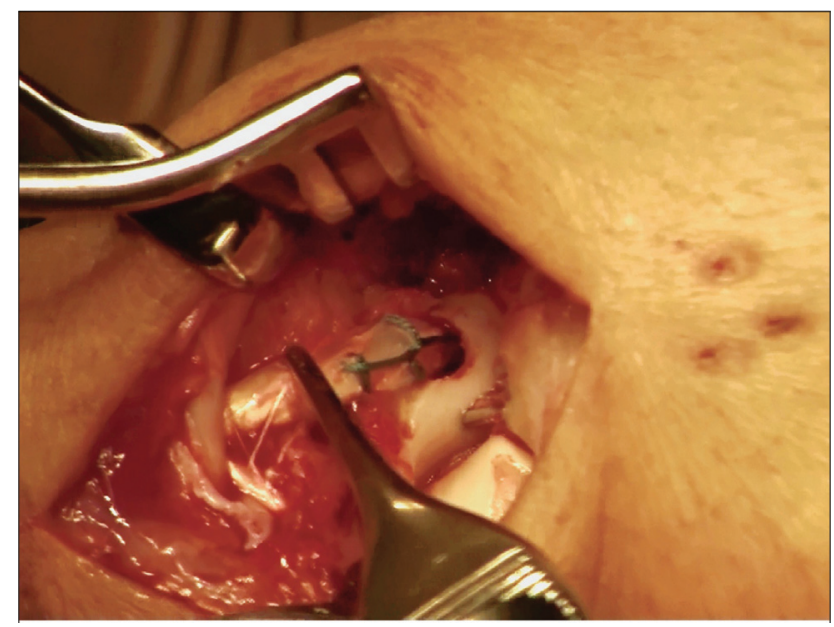

Fig. 6 Gliding of extensor carpi radialis longus is tested by pulling on the tendon dorsally.

did not have chondromalacia in the radiocarpal and midcarpal joints. The mean period from arthroscopy to Dynadesis was 5 months (range: 1-10 months). We excluded patients with acute wrist trauma, previous surgeries, fractures about the wrist joint, static deformity of the scaphoid, neuromuscular imbalances of the upper limb, or constitutional disorders.

Twenty patients ( 21 wrists) treated with the Dynadesis procedure reached a minimum follow-up of 20 years (range: 20-27). There were 15 females and five males, and the dominant hand was involved in 12 patients. The average age at the time of Dynadesis was 42 years. Geissler ${ }^{35}$ classifications of the SLIL tears were as follows: three Grade II, 16 Grade III ( - Fig. 7), and two Grade IV ( - Fig. 8) ligament injuries. All patients were classified as Garcia-Elias stage III. ${ }^{3}$ Average time to return to work and normal activities was five months (1-9 months) after Dynadesis. Clinical assessments included questionnaires, goniometric wrist range of motion (verified on X-rays), grip strength measurements (Jamar dynamometer), and seven view X-ray evaluations. An independent examiner performed final follow-up evaluations and analyzed X-rays. Institutional Review Board approval was obtained.

\section{Results}

Twenty-plus years after Dynadesis, the average grip strength increased by $8 \mathrm{~kg}$ (36\% improvement). Average wrist flexion
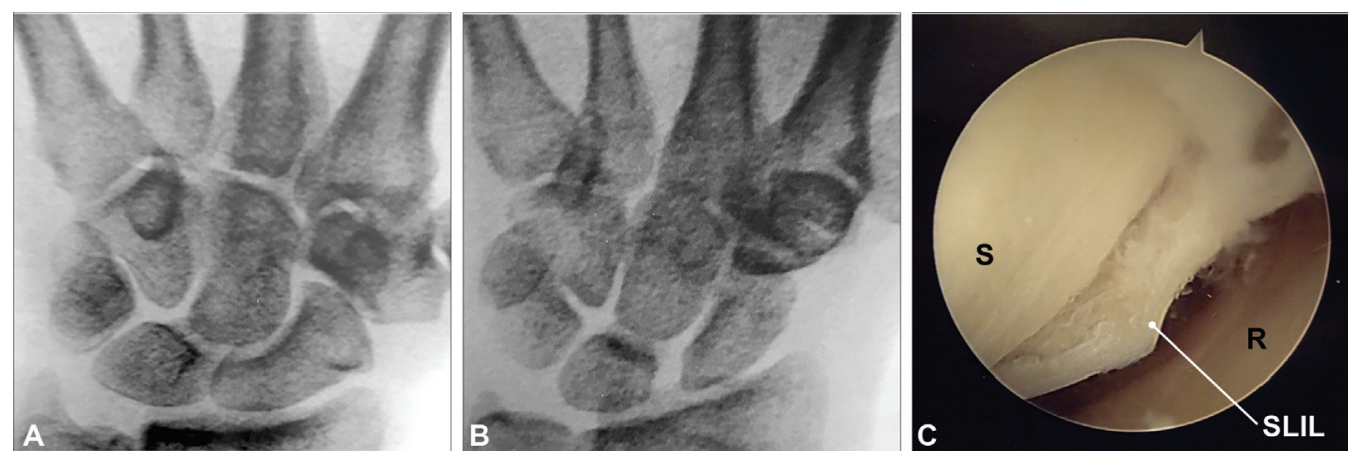

Fig. 7 Wrist X-rays and arthroscopy. (A) Resting PA wrist X-ray: normal scapholunate interval. (B) Clenched fist PA wrist X-ray: abnormal scapholunate interval. (C) Arthroscopy of radiocarpal joint of the wrist. R, radius; S, scaphoid; SLIL, scapholunate interosseous ligament. 

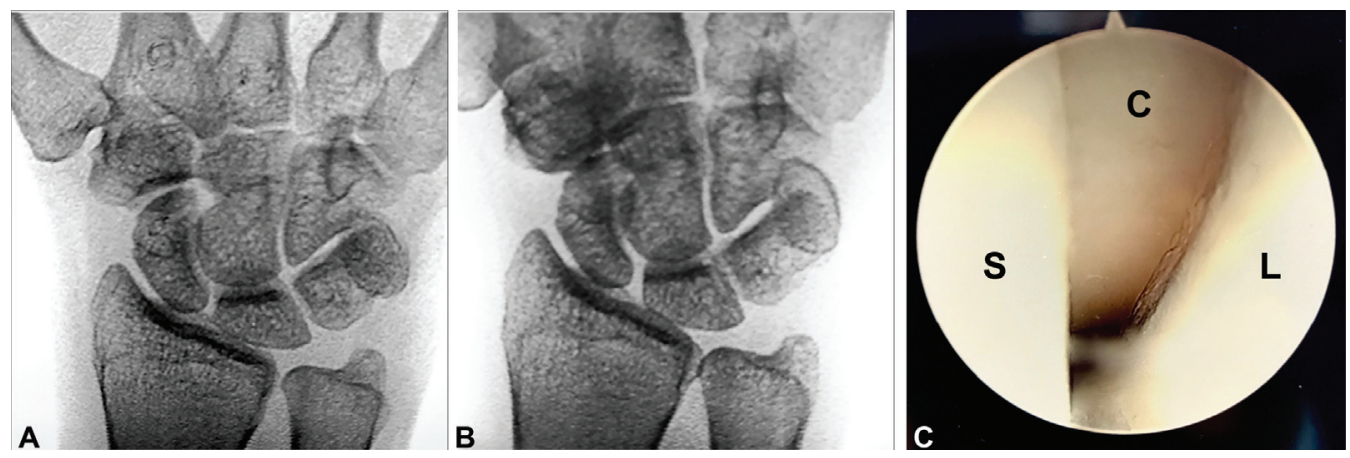

Fig. 8 Wrist X-rays and arthroscopy. (A) Resting PA wrist X-ray: normal scapholunate interval. (B): Clenched fist PA wrist X-ray: abnormal scapholunate interval. (C) Arthroscopy of radiocarpal joint of the wrist. C, capitate; L, lunate; S, scaphoid.

Table 1 Comparison of average, active wrist range of motion, pain, and grip strength preoperatively and $20+$ years after Dynadesis

\begin{tabular}{|l|l|l|l|l|l|l|}
\hline \multicolumn{7}{|c|}{ Comparison of preoperative and 20-year results } \\
\hline & Flex & Ext & RD & UD & Pain $^{\text {a }}$ & Grip \\
\hline Preoperative & $62^{\circ}$ & $51^{\circ}$ & $18^{\circ}$ & $29^{\circ}$ & 3.9 & $22 \mathrm{~kg}$ \\
\hline $\begin{array}{l}20+\text { years } \\
\text { after Dynadesis }\end{array}$ & $55^{\circ}$ & $55^{\circ}$ & $16^{\circ}$ & $33^{\circ}$ & 0.4 & $30 \mathrm{~kg}$ \\
\hline Change & $\downarrow 7^{\circ}$ & $\uparrow 4^{\circ}$ & $\downarrow 2^{\circ}$ & $\uparrow 4^{\circ}$ & $\downarrow 90 \%$ & $\uparrow 8 \mathrm{~kg}$ \\
\hline
\end{tabular}

Abbreviations: Ext, extension; Flex, flexion; RD, radial deviation; UD, ulnar deviation.

aPain measured from 0 to 5 on a visual analogue scale.

decreased $7^{\circ}$, while average wrist extension increased $4^{\circ}$. Before surgery, 95\% reported moderate-to-severe pain, and $20+$ years after Dynadesis, $76 \%$ reported no wrist pain (-Table 1). The Mayo wrist score ${ }^{37}$ (MWS) was used to assess outcomes. Because all but three patients had retired at final follow-up, the patients' work status at retirement was used to determine their functional status on the MWS. The average MWS score was 89 , with $76 \%$ of wrists in the excellent category. One patient with an ongoing workers compensation claim rated poor on the MWS ( - Table 2, wrist 12) but would recommend the procedure to someone else with the same problem. - Table $\mathbf{2}$ is a summary of the 20year results for each patient.

Comparisons were made of the preoperative X-rays and Xrays 20 years after Dynadesis. Scapholunate step-off, carpal height index, capitate-lunate angle, major arc, minor arc, ulnar variance, and the lunate-triquetrum gap remained essentially unchanged. A normal SL angle is a prerequisite for the diagnosis of DSI. The average preoperative and postoperative scapholunate angles were within normal limits $\left(58.2^{\circ}\right.$ and $\left.49.3^{\circ}\right)(p=0.035)$. The average scapholunate distance measured on a PA clenched-fist view decreased from $3.5 \mathrm{~mm}$ preoperatively to $2.4 \mathrm{~mm}(p=0.028) 20+$ years later. While this is a statistically significant decrease in the SL gap, the case numbers were small, and measurements from a 2D X-ray may not be as accurate as 3D studies. ${ }^{21}$

At the final evaluation, the average age of our patients was 62 years old. Age-related maladies plague any long-term study and were present in our patients. X-rays documented six patients with pan-trapezium arthritis (no pain: 4, postactivity discomfort: 2 ). One patient had bilateral 1st carpometacarpal arthritis with mild postactivity ache. Scaphotrapezio-trapezoid arthritis was observed in two wrists, both of which reported no pain. None of the patients with agerelated arthritis felt their conditions warranted treatment.

One patient fell onto her outstretched hand 2 months after her pins were removed and fractured through the drill hole in her distal scaphoid. She was immobilized in a thumb spica cast for 6 weeks and did not require further treatment. Twenty years later, she had no pain, her grip strength was $33 \mathrm{~kg}$, the wrist flexion-extension arc was $121^{\circ}$, and she scored 100 on the MWS ( - Table 2, wrist 8), even though her fracture had not united (-Fig. 9).

Most importantly, 20+ years after Dynadesis, wrist X-rays showed no evidence of increased subchondral ossification in the scaphoid fossa of the distal radius or reduction in the scaphoradial joint space in any of the cases. The drill hole remained open, with ossification of its walls, indicating that the ECRL remained functional, gliding through the drill hole in the scaphoid (-Fig. 10).

\section{Discussion}

Injury to the scaphoid ligaments causes instability of varying degrees, resulting in eventual degenerative changes in the wrist joint. ${ }^{3,36}$ When the SLIL is deficient, an applied load flexes and pronates the scaphoid, which increases the moment arms of the ECRL and FCR tendons, turning them into deforming forces that flex the scaphoid even further- ${ }^{24,28,29}$ a vicious cycle that accelerates degenerative changes. ${ }^{1,5,21,27}$ Over time, the STT ligament complex stretches, resulting in greater instability. As a result of this instability, the contact centroid of the scaphoid displaces dorsally, reducing the scaphoid's contact surface on the radius ${ }^{27,38,39}$ and increasing the joint compressive forces. Dynadesis is a tendon transfer of one muscle to its antagonist muscle via a tension-locking mechanism. This construct counters the deforming forces on the scaphoid without the undesirable effect of limiting scaphoid motion. ${ }^{18}$ When the wrist is loaded, the dynamic stabilizers of the scaphoid are stimulated to respond within $20 \mathrm{msec}$ by mechanoreceptors in the SLIL. ${ }^{30}$ When the SLIL is torn, impulses from the mechanoreceptors are reduced, ${ }^{32}$ altering 
Table 2 Final evaluation data for each patient 20 to 27 years after Dynadesis

\begin{tabular}{|c|c|c|c|c|c|c|c|c|c|c|c|}
\hline \multicolumn{12}{|c|}{ 20-year results } \\
\hline Wrist & $\mathrm{F} / \mathrm{U}$ years & Age at $F / U$ & Sex & Grip kg & Flex & Ext & F/E ArC & UD & RD & Pain $^{\mathrm{a}}$ & MWS \\
\hline 1 & 20 & 60 & $\mathrm{~F}$ & 28 & $76^{\circ}$ & $65^{\circ}$ & $141^{\circ}$ & $34^{\circ}$ & $14^{\circ}$ & 0 & 100 \\
\hline 2 & 21 & 67 & $\mathrm{M}$ & 54 & $50^{\circ}$ & $46^{\circ}$ & $96^{\circ}$ & $31^{\circ}$ & $16^{\circ}$ & 0 & 90 \\
\hline 3 & 20 & 64 & $M$ & 46 & $33^{\circ}$ & $57^{\circ}$ & $90^{\circ}$ & $29^{\circ}$ & $10^{\circ}$ & 0 & 90 \\
\hline 4 & 20 & 63 & $F$ & 24 & $19^{\circ}$ & $50^{\circ}$ & $69^{\circ}$ & $30^{\circ}$ & $8^{\circ}$ & 0 & 90 \\
\hline 5 & 20 & 55 & $\mathrm{~F}$ & 12 & $34^{\circ}$ & $60^{\circ}$ & $94^{\circ}$ & $32^{\circ}$ & $8^{\circ}$ & 1 & 70 \\
\hline 6 & 20 & 61 & $\mathrm{~F}$ & 16 & $20^{\circ}$ & $50^{\circ}$ & $70^{\circ}$ & $26^{\circ}$ & $18^{\circ}$ & 1 & 75 \\
\hline 7 & 20 & 57 & $\mathrm{M}$ & 32 & $30^{\circ}$ & $48^{\circ}$ & $78^{\circ}$ & $36^{\circ}$ & $6^{\circ}$ & 0 & 65 \\
\hline 8 & 20 & 64 & $\mathrm{~F}$ & 33 & $65^{\circ}$ & $56^{\circ}$ & $121^{\circ}$ & $40^{\circ}$ & $14^{\circ}$ & 0 & 100 \\
\hline 9 & 20 & 65 & $\mathrm{~F}$ & 32 & $88^{\circ}$ & $55^{\circ}$ & $143^{\circ}$ & $35^{\circ}$ & $15^{\circ}$ & 0 & 95 \\
\hline 10 & 21 & 63 & $\mathrm{~F}$ & 21 & $60^{\circ}$ & $40^{\circ}$ & $100^{\circ}$ & $30^{\circ}$ & $25^{\circ}$ & 1 & 100 \\
\hline 11 & 21 & 67 & $\mathrm{~F}$ & 30 & $60^{\circ}$ & $60^{\circ}$ & $120^{\circ}$ & $31^{\circ}$ & $13^{\circ}$ & 0 & 100 \\
\hline 12 & 22 & 54 & $\mathrm{~F}$ & 15 & $31^{\circ}$ & $53^{\circ}$ & $84^{\circ}$ & $32^{\circ}$ & $17^{\circ}$ & 5 & 50 \\
\hline 13 & 27 & 64 & $\mathrm{M}$ & 49 & $89^{\circ}$ & $62^{\circ}$ & $151^{\circ}$ & $45^{\circ}$ & $20^{\circ}$ & 0 & 100 \\
\hline 14 & 20 & 58 & $M$ & 33 & $45^{\circ}$ & $52^{\circ}$ & $97^{\circ}$ & $36^{\circ}$ & $10^{\circ}$ & 0 & 80 \\
\hline 15 & 20 & 62 & $\mathrm{~F}$ & 28 & $39^{\circ}$ & $58^{\circ}$ & $97^{\circ}$ & $24^{\circ}$ & $11^{\circ}$ & 1 & 100 \\
\hline 16 & 20 & 64 & $\mathrm{~F}$ & 35 & $70^{\circ}$ & $58^{\circ}$ & $128^{\circ}$ & $36^{\circ}$ & $32^{\circ}$ & 0 & 95 \\
\hline 17 & 21 & 64 & $\mathrm{~F}$ & 34 & $65^{\circ}$ & $55^{\circ}$ & $120^{\circ}$ & $36^{\circ}$ & $28^{\circ}$ & 0 & 95 \\
\hline 18 & 20 & 61 & $\mathrm{~F}$ & 30 & $90^{\circ}$ & $55^{\circ}$ & $145^{\circ}$ & $37^{\circ}$ & $17^{\circ}$ & 0 & 100 \\
\hline 19 & 22 & 61 & $\mathrm{~F}$ & 24 & $60^{\circ}$ & $55^{\circ}$ & $115^{\circ}$ & $38^{\circ}$ & $18^{\circ}$ & 0 & 90 \\
\hline 20 & 20 & 55 & $\mathrm{~F}$ & 34 & $65^{\circ}$ & $66^{\circ}$ & $131^{\circ}$ & $32^{\circ}$ & $15^{\circ}$ & 0 & 100 \\
\hline 21 & 26 & 77 & $\mathrm{~F}$ & 15 & $70^{\circ}$ & $63^{\circ}$ & $133^{\circ}$ & $20^{\circ}$ & $15^{\circ}$ & 0 & 90 \\
\hline Mean & 21 & 62 & & 30 & $55^{\circ}$ & $55^{\circ}$ & $111^{\circ}$ & $33^{\circ}$ & $16^{\circ}$ & 0.4 & 89 \\
\hline Max & 27 & 77 & & 54 & $90^{\circ}$ & $66^{\circ}$ & $151^{\circ}$ & $45^{\circ}$ & $32^{\circ}$ & 5 & 100 \\
\hline Min & 20 & 54 & & 12 & $19^{\circ}$ & $40^{\circ}$ & $69^{\circ}$ & $20^{\circ}$ & $6^{\circ}$ & 0 & 50 \\
\hline
\end{tabular}

Abbreviations: Ext, wrist extension; F, female; Flex, wrist flexion; F/E arc, flexion/extension arc; F/U, follow-up; M, male; MWS, Mayo wrist score; RD, radial deviation; UD, ulnar deviation.

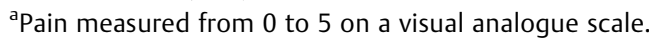

the response of the dynamic stabilizers. ${ }^{29,31}$ However, in Dynadesis, the reduced impulses are compensated for, as any motion of the scaphoid directly stimulates the ECRL, activating the construct. Furthermore, Dynadesis converts the ECRL to a direct scaphoid extender while simultaneously reducing the deforming moment arm of the $\mathrm{FCR}^{29}$ and pre-
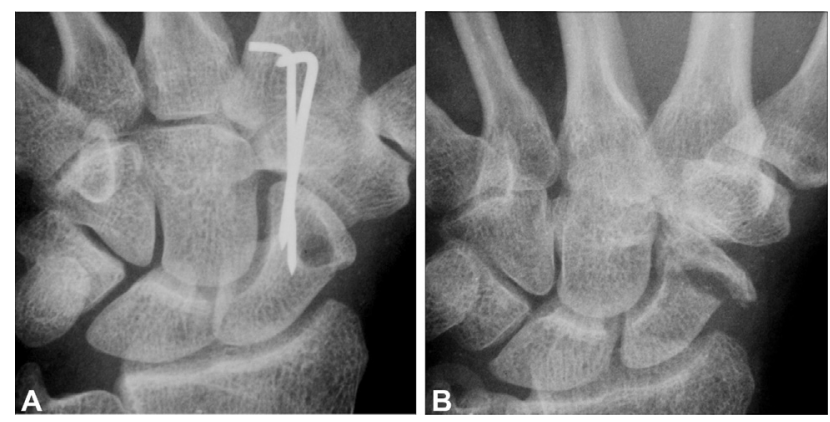

Fig. 9 Drill hole fracture. (A) Postoperative PA wrist X-ray, K-wires in place. (B) PA wrist X-ray, clenched fist. Scaphoid fracture, nonunion, 20 years after surgery. vents the secondary stabilizers from stretching out. Support is given to the volar STT ligaments by anchoring the ECRL to the FCR. This construct places additional tension directly on the

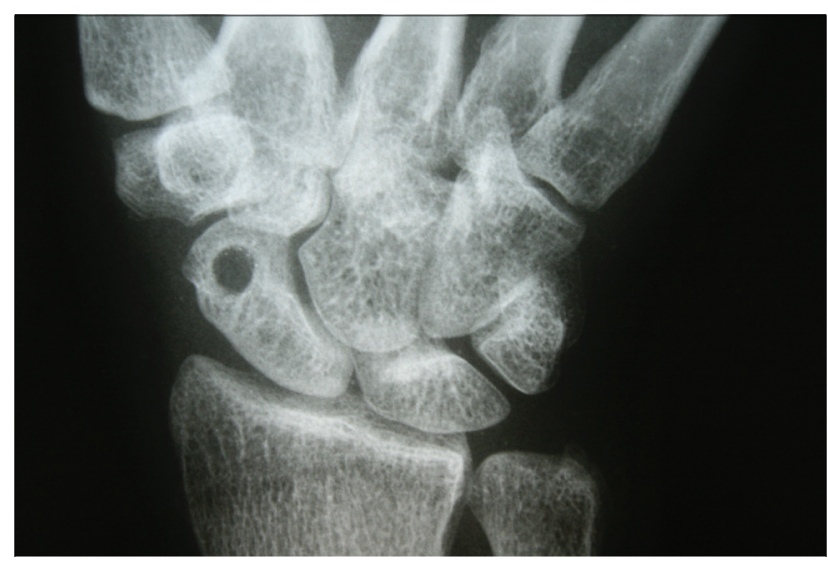

Fig. 10 Clenched fist, PA wrist $X$-ray, 20 years after Dynadesis. The drill hole wall is well-ossified, hole remains open, and scaphoradial joint space is well maintained. 
FCR tendon, creating a dynamic checkrein at the volar, distal scaphoid that is activated when the wrist is loaded and relaxed when the load is removed. We believe the construct does not stretch out over time due to the cocontraction of the ECRL and FCR muscles that dynamically tighten the construct every time the wrist is loaded. Additionally, these cocontractions prevent the secondary stabilizers (STT ligaments) from stretching.

Except for Dynadesis, none of the dynamic tendon transfer procedures address the passive, volar distal stabilizers of the scaphoid. Bleuler ${ }^{40}$ reported using the ECRL to treat static scaphoid instability. He followed 20 patients for four months and concluded that "... ECRL tenodesis is a reliable option for the successful treatment of dynamic scaphoid instability." Kaltenborn ${ }^{41}$ modified Bleuler's technique, treated 46 patients with a mixed bag of instabilities, and followed them for 2 years. Bleuler and Kaltenborn both refer to their procedures as a tenodesis. Peterson ${ }^{42}$ followed one patient with static scaphoid instability and a distal radius fracture for 6 months. His technique resembles the dorsal component of Dynadesis; however, he did not reinforce the passive volar scaphoid stabilizers. Recently, Stone ${ }^{43}$ reported a partial ECRL tendon transfer to the scaphoid but did not provide case numbers or follow-up data. He also refers to the procedure as a tenodesis and performs it in the same setting as the arthroscopy when some patients improve from arthroscopic debridement alone. ${ }^{44}$ Brunelli ${ }^{45}$ reports using the extensor carpi radialis brevis to treat one patient with a static deformity. He followed the patient for 8 months. In a meta-analysis of the literature for SLIL injuries, Montgomery ${ }^{46}$ found that the "optimal surgical technique remains unclear" due to poor patient selection and lack of heterogeneity of the procedures. We have not found any long-term reports of dynamic treatment of DSI in comparison to Dynadesis.

DSI is often misdiagnosed as a wrist sprain ${ }^{20,47}$ and goes untreated $^{4}$ until pain with activity increases. Furthermore, it frequently afflicts young adults ${ }^{4,48}$ while they still have a long and productive life ahead of them. For this reason, treatment proposals should be studied for decades rather than months or years. Calls have been made to tailor the treatment to the stage of instability, ${ }^{3-6,26,46}$ yet that concept has not taken hold in the wider hand surgery community. Review of the literature reveals a general disregard for the stages of instability, incorrect nomenclature (i.e., using tenodesis and tendon transfer interchangeably) adds to the confusion and published research is glaringly deficient of long-term studies.

Correct patient selection is mandatory for successful outcomes of Dynadesis, and it involves the following: 1) A thorough clinical examination; 2) An X-ray series including the clenched-fist view; 3) a wrist arthroscopy that documents $\mathrm{DSI}^{20}$ and evaluates all joint surfaces and wrist ligaments. ${ }^{36,49}$ The present imaging technology cannot detect early or small areas of localized chondromalacia, ${ }^{50}$ making arthroscopy mandatory. Furthermore, the SL gap size on a clenched-fist X-ray does not always correspond to the extent of the SLIL tear. ${ }^{21}$ For example, - Fig. 8 shows a small gap on a clenched-fist X-ray, but an arthroscopy reveals a Geissler grade 4 SLIL tear. Furthermore, arthroscopy of the wrist for DSI also has therapeutic value. In a 2020 presentation to the American Society for Surgery of the Hand, Seradge ${ }^{51}$ reported 79 consecutive patients with DSI. Forty-three patients (54\%) remained asymptomatic after wrist arthroscopic debridement alone with an average follow-up of 6.3 years and a maximum follow-up of 11 years.

A limitation of this study is that all surgeries were performed at a single institution by a single surgeon.

This long-term follow-up study (20-27 years) of patients who were treated with Dynadesis confirms that the construct did not stretch out over time and provided a return to normal life without limitations from wrist pain or weakness. With carefully selected patients, Dynadesis provides a satisfactory long-term result for treating DSI without limiting scaphoid motion.

Conflict of Interest

None declared.

\section{Acknowledgement}

The authors would like to acknowledge Espanta Steppe for her assistance with the illustrations.

\section{References}

1 Watson H, Ottoni L, Pitts EC, Handal AG. Rotary subluxation of the scaphoid: a spectrum of instability. J Hand Surg [Br] 1993;18(01): 62-64

2 Dyer GSM. Predynamic scapholunate instability. J Hand Surg Am 2010;35(11):1879-1880

3 Garcia-Elias M, Lluch AL, Stanley JK. Three-ligament tenodesis for the treatment of scapholunate dissociation: indications and surgical technique. J Hand Surg Am 2006;31(01):125-134

4 Pappou IP, Basel J, Deal DN. Scapholunate ligament injuries: a review of current concepts. Hand (N Y) 2013;8(02):146-156

5 Wolfe SW. Scapholunate instability. J Am Soc Surg Hand 2001;1 (01):45-60

6 Seradge H, Baer C, Dalsimer D, Seradge A, Shafi RAR, Parker W. Treatment of dynamic scaphoid instability. J Trauma 2004;56(06): 1253-1260

7 Szabo RM, Slater RR Jr, Palumbo CF, Gerlach T. Dorsal intercarpal ligament capsulodesis for chronic, static scapholunate dissociation: clinical results. J Hand Surg Am 2002;27(06): 978-984

8 Kleinman WB, Carroll C IV. Scapho-trapezio-trapezoid arthrodesis for treatment of chronic static and dynamic scapho-lunate instability: a 10-year perspective on pitfalls and complications. J Hand Surg Am 1990;15(03):408-414

9 Moran SL, Cooney WP, Berger RA, Strickland J. Capsulodesis for the treatment of chronic scapholunate instability. J Hand Surg Am 2005;30(01):16-23

10 Blatt G. Capsulodesis in reconstructive hand surgery. Dorsal capsulodesis for the unstable scaphoid and volar capsulodesis following excision of the distal ulna. Hand Clin 1987;3(01): 81-102

11 Glickel SZ, Millender LH. Ligamentous reconstruction for chronic intercarpal instability. J Hand Surg Am 1984;9(04):514-525

12 Sousa M, Aido R, Freitas D, Trigueiros M, Lemos R, Silva C. Scapholunate ligament reconstruction using a flexor carpi radialis tendon graft. J Hand Surg Am 2014;39(08):1512-1516

13 Nienstedt F. Treatment of static scapholunate instability with modified Brunelli tenodesis: results over 10 years. J Hand Surg Am 2013;38(05):887-892 
14 Brunelli GA, Brunelli GR. A new technique to correct carpal instability with scaphoid rotary subluxation: a preliminary report. J Hand Surg Am 1995;20(3 Pt 2):S82-S85

15 Linscheid RL, Dobyns JH. Treatment of scapholunate dissociation. Rotatory subluxation of the scaphoid. Hand Clin 1992;8(04): 645-652

16 Horn S, Ruby LK. Attempted scapholunate arthrodesis for chronic scapholunate dissociation. J Hand Surg Am 1991;16(02):334-339

17 Tomaino MM, Miller RJ, Cole I, Burton RI. Scapholunate advanced collapse wrist: proximal row carpectomy or limited wrist arthrodesis with scaphoid excision? J Hand Surg Am 1994;19(01): 134-142

18 Viegas SF, Patterson RM, Peterson PD, et al. Evaluation of the biomechanical efficacy of limited intercarpal fusions for the treatment of scapho-lunate dissociation. J Hand Surg Am 1990; 15(01):120-128

19 Seradge H, Sterbank PT, Seradge E, Owens W. Segmental motion of the proximal carpal row: their global effect on the wrist motion. J Hand Surg Am 1990;15(02):236-239

20 Andersson JK. Treatment of scapholunate ligament injury: current concepts. EFORT Open Rev 2017;2(09):382-393

21 Short WH, Werner FW, Green JK, Masaoka S. Biomechanical evaluation of the ligamentous stabilizers of the scaphoid and lunate: part II. J Hand Surg Am 2005;30(01):24-34

22 Lee SK, Desai H, Silver B, Dhaliwal G, Paksima N. Comparison of radiographic stress views for scapholunate dynamic instability in a cadaver model. J Hand Surg Am 2011;36(07):1149-1157

23 Mayfield JK, Johnson RP, Kilcoyne RK. Carpal dislocations: pathomechanics and progressive perilunar instability. J Hand Surg Am 1980;5(03):226-241

24 Tang JB, Ryu J, Omokawa S, Wearden S. Wrist kinetics after scapholunate dissociation: the effect of scapholunate interosseous ligament injury and persistent scapholunate gaps. J Orthop Res 2002;20(02):215-221

25 Boabighi A, Kuhlmann JN, Kenesi C. The distal ligamentous complex of the scaphoid and the scapho-lunate ligament. An anatomic, histological and biomechanical study. J Hand Surg $[\mathrm{Br}]$ 1993;18(01):65-69

26 Gelberman RH, Cooney WP, Szabo RM. Carpal instability. J Bone Joint Surg 2000;82(04):578

27 Viegas SF, Tencer AF, Cantrell J, et al. Load transfer characteristics of the wrist. Part II. Perilunate instability. J Hand Surg Am 1987;12 (06):978-985

28 Salvà-Coll G, Garcia-Elias M, Llusá-Pérez M, Rodríguez-Baeza A. The role of the flexor carpi radialis muscle in scapholunate instability. J Hand Surg Am 2011;36(01):31-36

29 Tang JB, Ryu J, Kish V. Scapholunate interosseous ligament sectioning adversely affects excursions of radial wrist extensor and flexor tendons. J Hand Surg Am 1997;22(04):720-725

30 Hagert E, Persson JKE, Werner M, Ljung B-OO. Evidence of wrist proprioceptive reflexes elicited after stimulation of the scapholunate interosseous ligament. J Hand Surg Am 2009;34(04): 642-651

31 Elsaftawy A. Radial wrist extensors as a dynamic stabilizers of scapholunate complex. Pol Przegl Chir 2013;85(08):452-459

32 Vekris MD, Mataliotakis GI, Beris AE. The scapholunate interosseous ligament afferent proprioceptive pathway: a human in vivo experimental study. J Hand Surg Am 2011;36(01):37-46

33 Garcia-Elias M, Lluch A. Wrist instabilities, misalignments and dislocations. In: Wolfe S, Hotchkiss R, Pederson W, Kozin S, Cohen
MS, eds. Green's Operative Hand Surgery. 7th ed. Philadelphia, PA: Elsevier; 2017

34 Moritomo H, Viegas SF, Nakamura K, Dasilva MF, Patterson RM. The scaphotrapezio-trapezoidal joint. Part 1: an anatomic and radiographic study. J Hand Surg Am 2000;25(05):899-910

35 Geissler WB. Arthroscopic management of scapholunate instability. J Wrist Surg 2013;2(02):129-135

36 Haerle M, Wahegaonkar A, Garcia-Elias M, Bain G, Luchetti R. Part 1: definition and investigations Paper presented at: IFSSH Scientific Committee on Carpal Instability - Part I. May 2017;07(02): $12-22$

37 Amadio PC, Berquist TH, Smith DK, Ilstrup DM, Cooney WP III, Linscheid RL. Scaphoid malunion. J Hand Surg Am 1989;14(04): 679-687

38 Burgess RC. The effect of rotatory subluxation of the scaphoid on radio-scaphoid contact. J Hand Surg Am 1987;12(5 Pt 1):771-774

39 Blevens AD, Light TR, Jablonsky WS, et al. Radiocarpal articular contact characteristics with scaphoid instability. J Hand Surg Am 1989;14(05):781-790

40 Bleuler P, Shafighi M, Donati OF, Gurunluoglu R, Constantinescu MA. Dynamic repair of scapholunate dissociation with dorsal extensor carpi radialis longus tenodesis. J Hand Surg Am 2008;33 (02):281-284

41 Kaltenborn A, Hoffmann S, Settje A, Vogt PM, Gutcke A, Rüttermann M. Modified minimally invasive extensor carpi radialis longus tenodesis for scapholunate dissociation: a prospective observational study. BMC Musculoskelet Disord 2017;18(01):54

42 Peterson SL, Freeland AE. Scapholunate stabilization with dynamic extensor carpi radialis longus tendon transfer. J Hand Surg Am 2010;35(12):2093-2100

43 Stone A, Shahid Z, Agarwal S, Sarkhel T. Extensor carpi radialis longus tenodesis using a biotenodesis screw for treatment of symptomatic Geissler 2 scapholunate dissociation. J Hand Microsurg 2019;11(Suppl 1):S53-S58

44 Weiss A-PC, Sachar K, Glowacki KA. Arthroscopic debridement alone for intercarpal ligament tears. J Hand Surg Am 1997;22(02): 344-349

45 Brunelli F, Spalvieri C, Bremner-Smith A, Papalia I, Pivato G. [Dynamic correction of static scapholunate instability using an active tendon transfer of extensor brevi carpi radialis: preliminary report]. Chir Main 2004;23(05):249-253

46 Montgomery SJ, Rollick NJ, Kubik JF, Meldrum AR, White NJ. Surgical outcomes of chronic isolated scapholunate interosseous ligament injuries: a systematic review of 805 wrists. Can J Surg 2019;62(03):1-12

47 Jones WA. Beware the sprained wrist. The incidence and diagnosis of scapholunate instability. J Bone Joint Surg Br 1988;70(02): 293-297

48 Rettig AC. Epidemiology of hand and wrist injuries in sports. Clin Sports Med 1998;17(03):401-406

49 Cooney WP. Evaluation of chronic wrist pain by arthrography, arthroscopy, and arthrotomy. J Hand Surg Am 1993;18(05): 815-822

50 Harley BJ, Werner FW, Boles SD, Palmer AK. Arthroscopic resection of arthrosis of the proximal hamate: a clinical and biomechanical study. J Hand Surg Am 2004;29(04):661-667

51 Seradge H, Parker W, Baer C, Steppe KR, Steppe KA. Incidence of open surgery in treatment of chronic scaphoid instability. Paper presented at: 75th Annual Meeting of the American Society for Surgery of the Hand; October 1-3, 2020;San Antonio, TX 